\title{
INSTRUMENTATION OF CURVED CANALS: A REVIEW
}

T. Senthil Kumar, Madhu Kiran, Prashant Tripathi, Sreenivasa Murthy

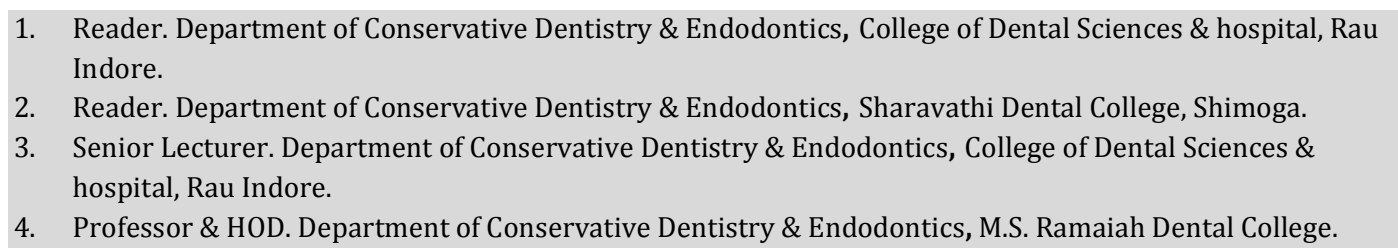

\section{CORRESPONDING AUTHOR}

Dr. T. Senthil Kumar,

B-5,115/2,S.V.Innovations,B.Narayanapura,

K.R.Puram hobli, Bangalore-16.

E-mail: drsenthil_kumar777@yahoo.com

Ph: 00919880311374

INTRODUCTION: Nature seldom draws a straight line. Nowhere is this more apparent than in the anatomy of teeth roots and root canal systems of human teeth. Even teeth with straight roots can harbor severely curved canals. Canal shaping is a critical aspect of endodontic treatment because it influences the outcome of the subsequent phases of canal irrigation and filling and the success of the treatment itself. In fact, curved canals are the most common endodontic complexity ${ }^{1}$.

The need for some manner of root canal preparation prior to root canal filling has long been recognized as an essential step in endodontic treatment. Concepts concerning the role and purpose of this canal preparation, however, have differed remarkably at different times in the development of endodontics and in the hands of different practitioners ${ }^{2}$.

Initially, root canals were manipulated primarily to allow placement of intracanal medicaments, with little attempt to remove completely the organic contents of the root canal system. In spite of elaborate modifications over the years, many methods of preparing root canals mechanically still fail to cleanse root canal systems effectively. In time, the concept of modifying root canal preparations to facilitate the placement of root canal fillings became part of the accepted endodontic practice, but the methods employed for these procedures remained, for the most part, unrelated both to the true anatomy of root canal systems and to the physical nature of the materials with which the root canals were presumed to be filled ${ }^{2}$.

PROBLEMS ENCOUNTERED IN CANAL PREPARATION: The most frequent problems occurring in canal preparation are root perforation, ledging and instrument breakage. Although any of these conditions may happen to even the best operator, it is important to develop the ability to keep such occurrences to an absolute minimum ${ }^{3}$.

These problems usually occur as a result of forcing and driving the instrument, over use of reaming action, and over reliance on chelating agents. To gain the full length of a sclerotic canal, it is often necessary to turn slightly, wiggle or otherwise plunge with the initial exploring instrument. However, once the full working length has been obtained and the canal shown to be patent, all other instruments must go easily to place with a straight, inserting action ${ }^{3}$.

Because the endodontic enlarging instruments resemble wood screws it is possible to force them into a canal and then by using rotating or reaming action, to gain deeper penetration. 
It is precisely at this point, when the file is pushed against a wall, that a deviation from the original canal shape results and then the operator creates his or her own shape when the files are forced into the dentin walls and bind, removal may result in breakage ${ }^{3}$.

If chelating agents are used heavily, the canal walls are softened and may be penetrated and altered to even a greater extent by forced files used with reaming action ${ }^{3}$.

Three important techniques may be utilized to prevent root perforation, ledging and instrument breakage. When files are precurved, original canal shape is more easily maintained, root perforation is lessened and reaming action must not be used. Incremental instrumentation allows instruments to be placed without forcing and the correct working length is gained without the need to use forceful reaming action. With the step or flared preparation, the smaller, more flexible files are used to the full working length; however, the larger more rigid instruments are kept away from the apex and confined to the straighter portions of the canal. Where they do not significantly alter original canal shape ${ }^{3}$.

\section{a) PRE-CURVING OF FILES:}

The most frequent problems occurring in canal preparation are root perforation, ledging and instrument breakage. Although any of these conditions may happen to even the best operator, it is important to develop the ability to keep such occurrences to an absolute minimum ${ }^{3}$.

These problems usually occur as a result of forcing and driving the instrument, over use of reaming action, and over reliance on chelating agents.

A.W.K.Chan et $\mathbf{a l}^{\mathbf{5}}$ determined the effects of hand instrumentation using traditional stainless steel K-files and nickel titanium K-files on the final shape of curved root canals. Both types of files transported the centre of the canals but the nickel-titanium instrument seemed to be safer because of the reduced amount of transportation towards the danger areas.

Philip J.Hankins et al ${ }^{6}$ compared the step back, balanced force and canal master instrumentation techniques using two different instrument types: Flex-R and CM.Canal angulation changes from pre operative to post rotary and post instrumentation were measured using the Schneider technique (ST) and the long axis technique (LAT).Rotary instrumentation resulted in an average loss of curvature of 2.37 degrees, as measured by the ST.The ST showed significant changes in the canal angulation between CM (-7.74 degrees) and SB (-5.28 degrees) groups. AT showed significant changes for $\mathrm{CM}$ versus $\mathrm{BF}$ and $\mathrm{SB}$, with a mean change in angulation of -7.69 degrees, -1.68 degrees and +0.1 degrees respectively.

During canal preparation in the course of endodontic treatment only the orifice is visible; the critical apical areas are never seen by the naked eye. Only the radiograph gives some scant knowledge about what happens near the tip of the root ${ }^{3}$.

In these more sharply curved canals, the shape changes more rapidly and may be undesirably altered, leading to potential failure. Virtually everyone who has performed endodontic treatment on a number of extremely curved canals has observed these changes, which are more than merely subtle3.

Benjamin Briseno marroquin ${ }^{7}$ introduced an anti-zipping preparation system (method and instrument) for curved root canals with which preparation effects such as "zips" and "elbowing" at the apical third can be easily eliminated.

Gary S.P.Cheung and Alex W.K.Chan ${ }^{8}$ compared the Excalibur hand piece with traditional K-files for their effectiveness in shaping curved root canals. They were instrumented 
either with Excalibur according to the manufacturer's instructions or manually using the stepdown technique. The cross sectional shapes of the root canal at the apical, midroot and coronal levels were digitized before and after instrumentation. The results showed that the methods removed similar amount of dentin at all three levels. Although the Excalibur hand piece had a slight tendency to straighten the canal more than hand instrumentation, the difference was not significant.

There are few straight root canals. Frequently the curvature is to the buccal or lingual aspect so that the routine radiographs give the false impression of a relative straight canal. Palatal roots of maxillary molars often have a buccal curve, whereas maxillary lateral and mandibular incisors may have lingual curves that do not appear on the radiograph because the curve is perpendicular to the film ${ }^{3}$.

In addition, as a result of eccentric dentin deposition or debris within the canal, the canal walls may have irregularities projections or other obstructions. When straight instruments are placed in such canals, they are stopped by the obstructions. If the file handle is rotated at this point, it will merely drive the tip of the instrument deeper into the impediment and create a ledge ${ }^{3}$.

For these reasons it is best to enter canals only with files that have been precurved. When this is done, the file will have a better chance to traverse any canal curvatures. Also, if an obstruction is encountered, rotation of the handle will allow the tip to slide off and continue toward the apex ${ }^{3}$.

There are two types of precurving. One is placing an extremely sharp curve near the tip of the instrument. This is used when the preparative radiograph discloses a sharp apical dilacerations or when an obstruction is encountered. The degree of curvature to give the instrument may be estimated by holding the file over the preoperative radiograph and increasing the curvature until the configurations of the file and the canal match. To avoid an obstruction, a short, sharper curve of approximately 30 to 40 degrees is usually sufficient. The other precurve is gradual for the entire length of the flutes and is to be used in all other cases ${ }^{3}$.

The sharper precurve is used when attempting to bypass a ledge from previous canal preparation and or filling. When the dentist is retreating a failing case that had short and straight canal fillings, the only hope for non-surgical success is to reach the area of apical foramen. Once the old fillings are removed or dissolved, using a straight file will merely continue the misdirection of the preparation. However, the sharply precurved instrument might be able to locate the correct direction and reclaim the case ${ }^{3}$.

The curving may be imparted by drawing the instrument across a metal ruler, cotton pliers, or other sterile instrument. The instrument must be resterilised before use if any nonsterile agent was used to give the curve ${ }^{3}$.

Once the precurved file is placed into the canal, there is no way to tell in which direction the curve bends. To avoid this problem the stop may be altered with a nick or flat end to indicate the curve. A rubber stop with a teardrop shape may be used with the point showing the correct direction of the curve ${ }^{3}$.

The use of reverse flaring greatly enhances the ease by which precurved files may be inserted. The wider orifice will accommodate these instruments and prevent them from curling back onto themselves, which might happen when they are placed into narrow sites. Particularly in molar canals without the orifice being widened, it is difficult to keep the curve intact, especially the type that is a sharp curve ${ }^{3}$. 
b) Incremental instrumentation: The development of standardization increased the number of instrument diameters available for use, particularly in the smaller sizes. Therefore it became easier to place instruments to their correct working length, without the forcing or rotation that might cause breakage or prepare a false canal ${ }^{3}$.

Even so, in difficult cases even the small increment of $0.05 \mathrm{~mm}$ between instruments is still too great. A new instrument may not achieve the same position that the previously used smaller size reached. The solution to this problem is to create new increments between the established widths by cutting off a portion of the file tip, thus making it slightly wider in diameter ${ }^{3}$.

The rule relating to standardized instruments has established that the distance between D0 and D16 (the minimum length of the flutes) is $16 \mathrm{~mm}$ and that the difference in diameter between these two points is $.32 \mathrm{~mm}$ or $.02 \mathrm{~mm}$ of width per millimeter of length. Therefore, if a $1 \mathrm{~mm}$ segment is clipped from a size 10 file, the instrument becomes a size $12,1 \mathrm{~mm}$ from a size 25 file is a size 27 , and so forth ${ }^{3}$.

To utilize this procedure in difficult cases, after using the IAF in small, curved canals (usually size 10 or 15 ), the dentist should routinely trim $1 \mathrm{~mm}$ from it (creating a size 12 or 17 , respectively) before going to the next larger size. Because the cutting the shaft imparts a flat tip a metal nail file is used to smooth the end and reestablish a bevel after the removal of any segment ${ }^{3}$.

In some extremely curved and very sclerotic canals, cutting off one full millimeter to widen the instrument by $0.02 \mathrm{~mm}$ is too great to enable the dentist to reach the full working length easily without forcing and / or using reaming action and thus potentially altering original canal shape. Therefore in these cases only $0.5 \mathrm{~mm}$ segments are trimmed, increasing the instrument width by $0.01 \mathrm{~mm}$ and making a size 10 into a size 11 , a size 15 into a size 16,and so forth. Again, the nail file is used after each trimming. In these extremely difficult cases successive trimming of $0.5 \mathrm{~mm}$ and $1 \mathrm{~mm}$ segments can bring an initial instrument to a greater width than the next standard size. This works well, because of the possible variance in even quality - controlled instruments, to avoid the use of an incorrectly sized or marked second file, which might ledge a well-started difficult case. Unfortunately these successive cuttings reduce flute length; after 5 to $7 \mathrm{~mm}$ is removed, too little cutting distance remains to be effective, and the remaining instrument has lost much of its flexibility 3 .

c) Instruments with intermediate sizes: Because of the logic for making intermediate-sized instruments when treating the more difficult canals, several companies have manufactured file systems that incorporate aspects of this technique ${ }^{3}$.

Golden mediums made by Maillefer, are the files made according to the standardized principles in the sizes that until now available in size no. 12,17, 22, 27, 32 and 37 . They were manufactured so that the clinician did not have to clip files but instead had a readily available size in between the normally manufactured sizes ${ }^{3}$.

The fallacy for this file system is that every file is not, nor need be exactly what the Do designation indicates but the standard allows a deviation of up to $0.02 \mathrm{~mm}$ to still be acceptable. Therefore a size no. 10 instrument may be as small as 0.08 or as large as $0.12 \mathrm{~mm}$ at Do and still be acceptable. This explains why sometimes the clinician may have great difficulty going from one size to the next larger in narrow, curved canals without using intermediate sizes ${ }^{3}$.

By the same token, the intermediate sizes may almost overlap or even slightly overlap the standard sizes, even if manufactured with tight controls. Size no. 10 files may easily be size no. 11 and the size 12 (medium file) only slightly wider, perhaps no 11.5. This would allow the 
medium file to go quite easily after the supposed size no. 10. However, what if the no 15 file was really size no 16.5 ? It probably still would not go easily into a small curved canal. What is available for the clinician to do? The answer is to clip the no $12^{3}$.

The only way to ensure that one size file is slightly larger than the previous file used to clip the file. Any other method chances an error, no matter how tightly the manufacturing process is controlled 3 .

Another file system is the series 29. Schilder has stated that even with the increased number of small-sized files introduced by the standardized system (sizes no.08, 10 and 15) as opposed to the old 1 to 12 system (old no. 1 was usually slightly larger then the new no. 10; old no 2 was usually intermediate between new no 15 and no 20), more small sizes were needed in difficult canals. Schilder proposed that instead of increasing each small file by $0.05 \mathrm{~mm}$ between sizes, they should be increased by $29 \%{ }^{3}$.

Therefore, if size no. 10 (Do to be $0.01 \mathrm{~mm}$ ) were to be established as a key size, the file smaller than it would be $0.077 \mathrm{~mm}$ at Do and the file larger would be $0.129 \mathrm{~mm}$ at Do. This system works well in these small sizes, but the difficulties begin after the fourth step, when one must go from a Do of $0.216 \mathrm{~mm}$ to that of $0.279 \mathrm{~mm}$ and then upto $0.360 \mathrm{~mm}$. This is not possible in a difficult, small and curved canal. The only available solution, again, would be to clip the file to utilize intermediate sizes 3 .

\section{PREPARATION IN EXTREMELY CURVED CANALS}

Many methods and techniques of canal preparation work well in the larger and relatively straight canals. However when the canal curvature reaches 30 degrees or more, the complexity of the case increases markedly, and the techniques that render good results in the simpler cases may or may not be successful ${ }^{3}$.

\section{a) Canal characteristics not seen on routine radiographs}

\begin{tabular}{|l|l|l|}
\hline \multicolumn{1}{|l|}{ TOOTH } & \multicolumn{1}{|l|}{$\begin{array}{l}\text { BUCCOLINGUAL ROOT } \\
\text { CURVATURES }\end{array}$} & $\begin{array}{l}\text { BUCCOLINGUAL CANAL } \\
\text { EXITING }\end{array}$ \\
\hline MAXILLARY TEETH & Very rare \\
Central & $\begin{array}{l}\text { Distolingual common } \\
\text { Rare }\end{array}$ & $\begin{array}{l}\text { Short to buccal } \\
\text { To distolingual }\end{array}$ \\
Lateral & $\begin{array}{l}\text { Succal possible } \\
\text { Buccal or palatal possible } \\
\text { Cuspid }\end{array}$ & $\begin{array}{l}\text { Short to buccal } \\
\text { - }\end{array}$ \\
First bicuspid & \begin{tabular}{l} 
Shal \\
\hline
\end{tabular}
\end{tabular}




\section{REVIEW ARTICLE}

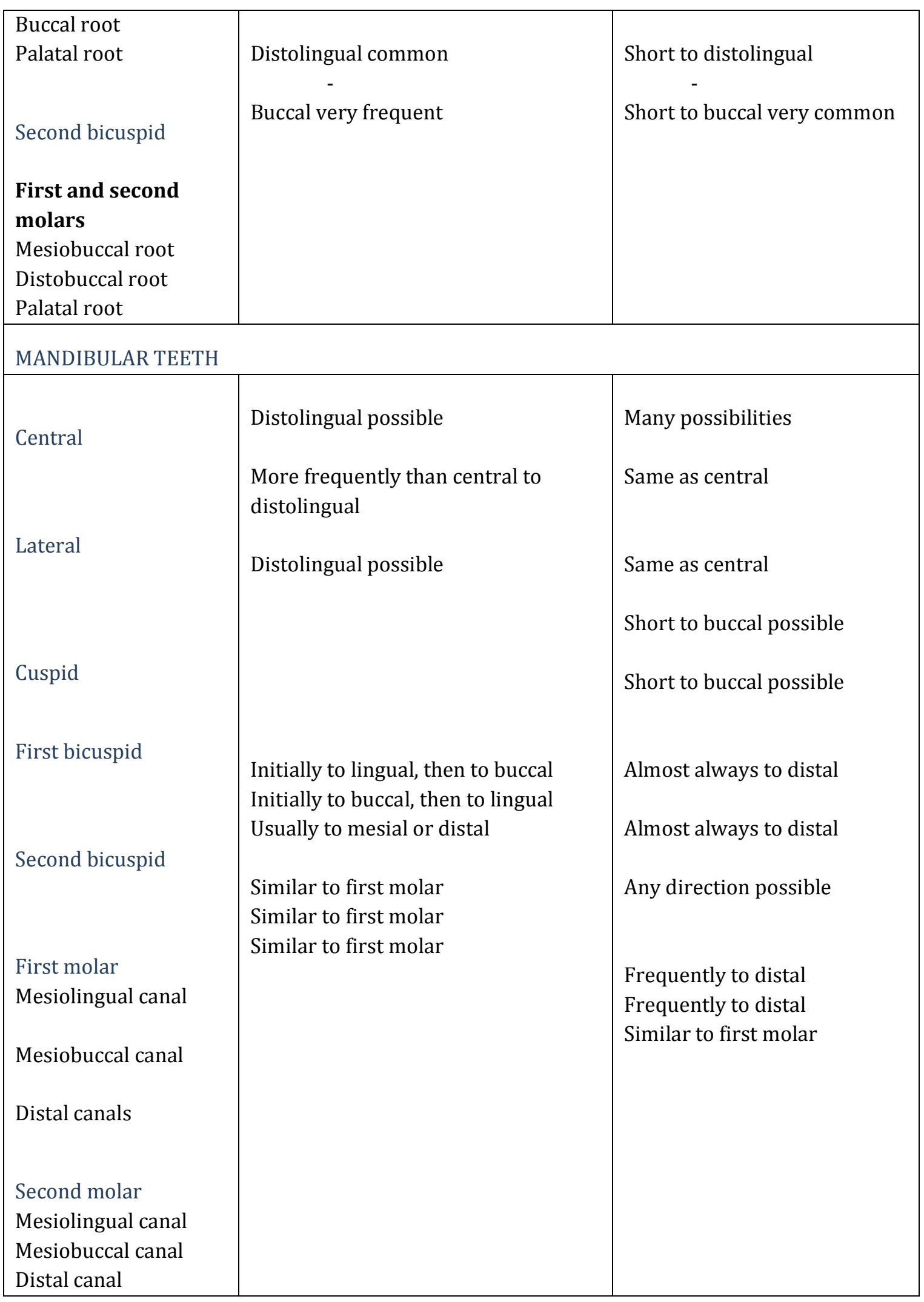




\section{b) Determination of canal curvature:}

Before the initiation of treatment, an estimate should be made as to the degree of curvature of the canals to be treated. As described originally by Schneider and then Jungman et.al, the method for making this determination is quite simple. In most instances a radiograph will indicate that the curved canal has two segments, One extending from the floor of the chamber down the long axis of much of the coronal two thirds of the root and the second from the apex of the root extending back to the occlusal through the apical third of the root. These two lines will intersect and form four angles. The interior angle is the estimate of the degree of canal curvature ${ }^{3}$.

Because it is calculated from a two-dimensional radiograph, this is merely an estimate and determines the mesiodistal curvature only without taking into consideration any buccolingual curvature. The operator should be aware of the teeth that typically have buccolingual curvature and take this into consideration ${ }^{3}$.

The operator should also be aware of the shape of the initial files after they are removed from the tooth. Often some small, abrupt or unnoticed curve will be indicated by an unusual shape in the file that corresponds to the true curvature within the canal. It is important to utilize this knowledge when further preparation is performed ${ }^{3}$.

C) Need for remeasurement when preparing curved canals: As enlarging instruments are placed in and removed from curved canals, the flutes cut more deeply into the portion of inner surface of the bend. As preparation progresses, this tends to straighten out the canal to some degrees. In addition, through removal of this portion of the curve, the working length is effectively decreased because less length of file is required to go around the bend. Continuing to file at the original length will lead to over instrumentation and resultant periapical tenderness. Since it is so important to have an apical matrix to pack filling material against, failure to allow for this decrease in working length will complicate the filling of the canal. Therefore, when curved canals are enlarged, radiographs to check for a new working length should be taken for every three increments of file width (eg., if a size 15 file is the first instrument used, at size 25 a new radiograph is taken) and any decrease in working length is calculated 3 .

d) Observation that canal shape changes: It is important to retain original canal shape. However, it has been noticed for many years that the original canal shape may be ever so slightly altered with each file in the curved canal. Individually there is only a slight alteration between one file size and the next larger instrument, but the net change can be quite drastic ${ }^{3}$.

During canal preparation in the course of endodontic treatment only the orifice is visible; the critical apical areas are never seen by the naked eye. Only the radiograph gives some scant knowledge about what happens near the tip of the root ${ }^{3}$.

In these more sharply curved canals, the shape changes more rapidly and may be undesirably altered, leading to potential failure. Virtually everyone who has performed endodontic treatment on a number of extremely curved canals has observed these changes, which are more than merely subtle3. 
e) Plastic block studies: In an attempt to clarify the actions of the instrument during canal preparation, acrylic blocks were prepared that would simulate intracanal treatment conditions and allow for complete visualization of the procedures. Small silver points were bent into a curve to simulate curved molar canals. The points were bent into a curve to simulate curved molar canals. The points were lubricated and placed in a mix of clear acrylic casting resin. When the plastic hardened, the points were removed and a canal was left in the block. The knoop hardness number of the blocks was 22, compared to 40 for dentin near the pulpal wall. According to Patterson, the number could be reduced to as low as 7 for dentin exposed to EDTA. The filing of the blocks felt similar to the filing of normal teeth ${ }^{3}$.

These blocks were distributed to a number of endodontists and general dentists who had considerable endodontic experience. The practitioners asked to prepare these canals in their routine manner. Some fascinating information was gleaned from analyzing these preparations. For years it had been thought that after the completion of preparation, the narrowest point of the canal was at the apex. This was taken for granted, because it had to be true if endodontic therapy were to experience predictable successes. However, whereas the widest portion of the canals prepared in the blocks was at the orifice, the narrowest site was never at the apex but was a few millimeters short of the apex ${ }^{3}$.

When the paper describing this study was reported by Weine, Kelly and Lio, many strongly doubted its authenticity. They could not accept that in a curved canal which could be completely visualized at all times and with the file seemingly guided, as it could never be in a true clinical case, it was impossible to gain a preparation narrowest at the apex. This cast considerable doubt over the veracity of the use of silver cones in curved canals because of the impossibility of the solid silver cone filling the biconcave shape. Even vertically or laterally condensed gutta-percha would have considerable problems here because a perfect funnel does not exist to allow for the packing down to the narrowest diameter at the tip ${ }^{3}$.

In actuality, a study by Guiterrez and Garcia 7 years earlier on extracted teeth had reported many of these same observations, particularly concerning the tip of the preparation. These authors had stated that an hourglass shape existed at that site, whereby the narrowest point was not at the tip of the preparation but was several millimeters coronal from the tip. This was emphatically verified by the plastic block studies ${ }^{3}$.

A further observation was made during the course of instrumentation in the curved plastic block canals. During insertion or withdrawal, each file attempted to straighten within the canal, whether precurved or not. Therefore the file rode the inner portion of the canal between the orifice and the narrowest point and the tip of the preparation. Between the narrowest point and the tip, each succeeding file went farther and further away from the inner portion of the curve. It was impossible to avoid this tendency, even with increased precurving or attempting to redirect the file visually, which could be done with transparent blocks but obviously not in a clinical case ${ }^{3}$.

Viewing the base of the blocks, one could see that which would conform to the apical foramen gave an interesting perspective. In none of these blocks were the sites of exiting round or even elliptical, but were teardrop shaped. The narrowest portion of the teardrop was where the initial smallest files exited from the block with the sharpest portion of the curvature. As each larger file exited farther and farther away from the inner portion of the curve, it widened the teardrop to that direction ${ }^{3}$. 
This situation may occur in a clinical case as well. When the radiograph is viewed, it might be assumed that the root tip had been perforated. In actuality, this is not a perforation but a slit or teardrop in the root tip. A perforation has two canals, the true canal and the false canal. in the apex that has been teardropped, the initial files made the curve but slightly overinstrumented the apex. Each succeeding file opened up slightly away from the curve, completing the error ${ }^{3}$.

Because of the teardrop shape, condensed gutta-percha might be able to seal such a shape, but only at the cost of an overfill, which will probably invoke a rather severe postoperative tenderness and cause problems in healing. In addition to those critics who assailed the original Weine, Kelly, and Lio paper as inaccurate, many attacked the use of the plastic block as a substitute for extracted teeth studies or actual clinical cases. This view has now been reversed 180 degrees, and the use of plastic blocks has become quite dominant. In fact if a new preparation technique or new endodontics instrument is introduced without an accompanying plastic block study, its veracity would remain in considerable question ${ }^{3}$.

f) Standard preparation in the sharply curved canal: Observations of block studies led to a number of interesting conclusions that were verified constantly, regardless of which operator was performing the preparations. The initial flexible files in a curved canal are able to make the curve and stay within the confines of the true canal. However, as the larger, less flexible instruments are used, they do more work on the inner portion of most of the canal and on the outer portion of the canal near the tip. The result is misshapen canal that is so difficult to fill. The site where the canal is the narrowest was called the ELBOW and the wider irregular area at the tip of the root was called the Zip. A cross sectional view of the tip is the teardrop shaped site, and a canal prepared in this manner is considered zipped 3 .

g) Applications on extracted teeth: To clarify their study further, Weine et al. Slightly over prepared canals in teeth where the apical foramen came off shorter than routine, which therefore could be prone to such preparations. The results of these preparations verified that the plastic block studies were accurate. Custom mounts were rephotographed in virtually the same position before, during and after preparation. The canals were prepared in a normal manner and the results analyzed. In these teeth the teardrop shapes at the apices were clearly demonstrable. Radiographs taken during the course of treatment verified the existence of the apical zip, elbow and hourglass shape. The correlation between these findings on extracted teeth and those of clinical cases in unfortunately true ${ }^{3}$.

h) Avoiding the apical zip and the elbow: Once these tendencies could be categorized and verified, potential solutions were devised. It seems obvious that in order to reduce the apical zip, we must prevent the file from opening at the apex ${ }^{3}$.

When the tip of the endodontic file is clipped to produce the intermediate sizes the diamond edged nail file is used to rebevel the tip of the instrument.

To avoid the apical zip, after the clip is made to gain an intermediate size, the nail file is dragged against the outer portion of the precurved endodontic file to remove the cutting flutes and thus produce a customized file - a file for that canal shape only. This should not be accomplished with a stone mounted in a hand piece. The distance between the elbow and the apex is estimated on the preoperative radiograph, and that length of flutes is removed from the outer surface of the endodontic instrument. If it were done on every instrument in the canal, there would be no preparation on the outer portion of the curve near the apex and the canal would be over prepared in the opposite direction ${ }^{3}$. 
The elimination of the elbow is much less difficult to accomplish. All that is needed is to provide adequate canal flaring. This opens up the bottleneck of the elbow and leads to a truly tapered canal, which can be filled by a multiplicity of techniques and materials ${ }^{3}$.

\section{III) STEP-BACK OR STEP-DOWN?}

Two approaches to debriding and shaping the canal have finally emerged: either starting at the apex with fine instruments and working one's way back up (or down) the canal with progressively larger instruments-the "step-back" or serial technique-or the opposite, starting at the cervical orifice with larger instruments and gradually progressing toward the apex with smaller and smaller instruments-the "step-down" technique, also called "crown -down" filing 4

Hybrid approaches have also developed out of the two methods. Starting coronally with larger instruments, often power driven, one works down the straight coronal portion of the canal with progressively smaller instruments-the step down approach. then, at this point, the procedure is reversed, starting at the apex with small instruments and gradually increasing in size as one works back up the canal-the step-back approach. This hybrid approach could be called, quite clumsily, the step-down-step back technique or "modified double-flared technique4."

Weine, Martin, Walton and Mullaney were early advocates of step-back, also called telescopic or serial root canal preparation. Designed to overcome instrument transportation in the apical third canal, as described earlier, it has proved quite successful.

\section{STEP-BACK PREPARATION AND CURVED CANALS:}

This method of preparation has been well described by Mullaney. His approach has been modified, however to deliver a continuing tapered preparation. Mullaney divided the step-back preparation into two phases. Phase I is the apical preparation starting at the apical constriction. Phase II is the preparation of the remainder of the canal, gradually stepping back while increasing in size. The completion of the preparation is the refining phase IIA and IIB to produce the continuing taper from apex to cervical ${ }^{4}$.

\section{STEP-BACK, STEP-BY-STEP--HAND INSTRUMENTATION:}

Phase I. To start phase I instrumentation, it must be assumed that the canal has been explored with a fine pathfinder or instrument and that the working length has been established-that is, the apical constriction identified. The first active instrument to be inserted should be a fine (No.08, 10,or 15) 0.02,tapered, stainless steel file, curved and coated with a lubricant, such as Gly-oxide, R.C.prep, file-Eze, glyde, K-Y Jelly, or liquid soap. The flexibility of nickel titanium does not lend itself to this pathfinding function in sizes smaller than no.154.

The motion of this instrument is "watch winding," two or three quarters-turns clockwisecounterclockwise and then retraction. On removal, the instrument is wiped clean, recurved, relubricated, and repositioned. "Watch winding" is then repeated. Remember that the instrument must be to full depth when the cutting action is made. This procedure is repeated until the instrument is loose in position. Then the next size $\mathrm{k}$ file is used-length established, precurved, lubricated, and positioned. Again, the watch-winding action and retraction are repeated. Very short $(1.0 \mathrm{~mm})$ filing strokes can be used at the apex. At the University of 
Tennessee, nickel titanium files were not curved and maintained the canal shape better than stainless steel ${ }^{4}$.

It is most important that a lubricant be used in this area. As berg and Buchanan pointed out, it is often fibrous pulp stumps, compacted into the constricture, that cause apical blockage. In very fine canals, the irrigant that will reach this area will be insufficient to dissolve tissue. Lubrication, on the other hand, emulsifies tissue, allowing instrument tips to macerate and remove this tissue. It is only later in canal filing that dentin chips pack apically, blocking the constriction. By then the apical area has been enlarged enough that sodium hypochlorite can reach the debris to douche it clear4.

By the time a size $25 \mathrm{~K}$ file has been used to full working length, phase I is complete. The 1.0 to $2.0 \mathrm{~mm}$ space back from the apical constriction should be clean of debris unless this area of the canal was large to begin with, as in a youngster. Then of course, larger instruments are used to start with4.

Using a number 25 file here as an example is not to imply that all canals should be shaped at the apical restriction only to size 25. Hawrish pointed out the apparent lack of interest in canal diameter versus the great interest in the proper canal length. Many, in fact most, canals should be enlarged beyond size 25 at the apical constriction in order to round out the preparation at this point and remove as much of the extraneous tissue, debris, and lateral canals as possible.

As stainless steel instruments become larger, they become stiffer. Metal "memory" plus stress on the instrument start its straightening. It will no longer stay curved and starts to dig, to zip the outside (convex) wall of the canal ${ }^{4}$.

Phase II. In a fine canal (and in this example), the step back process begins with a no.30 K-style file. Its working length is set $1 \mathrm{~mm}$ short of the full working length. It is Precurved, lubricated, carried down the canal to the new shortened depth, watch wound, and retracted. The same process is repeated until the No.30 is loose at this adjusted length. Recapitulation to full length with a No.25 file follows to ensure patency to the constriction. This is followed by copious irrigation before the next curved instrument is introduced. In this case, it is a No.35, again shortened by $1.0 \mathrm{~mm}$ from the No.30 is loose at this adjusted length.

Recapitulation to full length with a No.25 file follows to ensure patency to the constriction. This is followed by copious irrigation before next curved instrument is introduced. In this case, it is a no.30 $(2.0 \mathrm{~mm}$ from the apical no.25). It is curved, lubricated, watch wound, and retracted followed by recapitulation and irrigation ${ }^{4}$.

Thus, the preparation steps back up the canal $1 \mathrm{~mm}$ and one larger instrument at a time. When that portion of the canal is reached, usually the straight midcanal, where the instruments no longer fit tightly, then perimeter filing may begin, along with plenty of irrigation ${ }^{4}$.

It is at this point that Hedstroem files are most effective. They are much more aggressive rasps than the $\mathrm{K}$ files. The canal is shaped into the continuous taper so conducive to optimum obturation. Care must be taken to recapitulate between each instrument with the original No.25 file along with ample irrigation ${ }^{4}$.

The midcanal area is the region where reshaping can also be done with power-driven instruments: Gates-Glidden drills, starting with the smaller drills (Nos.1 and 2) and gradually increasing in size to No.4, 5, or 6.proper continuing taper is developed to finish Phase II A preparation. Gates-glidden drills must be used with great care because they tend to "screw" themselves into the canal, binding and then breaking4. 
To avoid this, it has been recommended that the larger sizes be run in reverse. But, unfortunately, they do not cut as well when reversed. A better suggestion is to lubricate the drill heavily with RC-Prep or Glyde, which "will prevent binding and the rapid advance problem." Lubrication also suspends the chips and allows for a better "feel" of the cutting as well as the first canal curvature. Used gates-Glidden drills are also less aggressive than new ones 4 .

Newer instruments with various tapers from 0.04 to $0.08 \mathrm{~mm} / \mathrm{mm}$ of taper are now available for this purpose as well and can be used as power-driven or hand instruments. With any of these power driven instruments, using them in a passive pecking motion will decrease the chances of binding or screwing into the canal ${ }^{4}$.

Refining phase IIB is a return to a size No.25 (or the last apical instrument used), smoothing all around the walls with vertical push-pull strokes, to perfect the taper from the apical constriction to the cervical canal orifice. In this case, a safe ended, non-cutting tip, Hedstroem file is the most efficient. It produces a good deal of dentin chips, however that must be broken up at the apex with a cutting tip K-file and then flushed out with abundant sodium hypochlorite ${ }^{4}$.

At this point, Buchanan recommended that sodium hypochlorite be left in place to the apex for 5 to 10 minutes. This is the only way in which the auxiliary canals can be cleaned. Hand powered gates-Glidden drills (handy gates) or light speed instruments may be used for this final finish, as well as the new hand piece orifice openers or gates-Glidden drills. Gutmann and Rauskin pointed out that the "final preparation should be an exact replica of the original canal configuration-shape, taper, and flow, only larger". So-called "coke-bottle" preparations should be avoided at all cost ${ }^{4}$.

MODIFIED STEP-BACK TECHNIQUE: One variation of the step-back technique is more traditional. The preparation is completed in the apical area, and then the step-back procedure begins 2 to $3 \mathrm{~mm}$ up the canal. This gives short, almost parallel retention form to receive the primary gutta-percha point when lateral condensation is being used to fill the canal. The guttapercha trial point should go fully to the constriction, and a slight tug-back should be felt when the point is removed (retention form). This shows that it fits tightly into the last 2 to $3 \mathrm{~mm}$ of the prepared canal 4 .

IV) STEP-DOWN TECHNIQUE-HAND INSTRUMENTATION: Initially, Marshall and Pappin advocated a "crown-down pressure-less preparation" in which Gates-Glidden drills and larger files are first used in the coronal two-thirds of the canals and progressively smaller files are used from the "crown down" until the desired length is reached. This has become known s the step-down or crown-down technique of cleaning and shaping

A primary purpose of this technique is to minimize or eliminate the amount of necrotic debris that could be extruded through the apical foramen during instrumentation. This would help prevent post-treatment discomfort, incomplete cleansing, and difficulty in achieving a biocompatible seal at the apical constriction ${ }^{4}$.

Step-down, step-by-step In this method, the access cavity is filled with sodium hypochlorite, and the first instrument is introduced into the canal. At this point, there is a divergence in technique dictated by the instrument design and the protocol for proceeding recommended by each instrument manufacturer. All of the directions, however, start with exploration of the canal with a fine, stainless steel, 0.2 taper (No.8, 10, 15, or 20 file, determined by the canal width), 
curved instrument. It is important that the canal be patent to the apical constriction before cleaning and shaping begin ${ }^{4}$.

Sometimes the chosen file will not reach the apical constriction, and one assumes that the file is binding at the apex. But, more often than not; the file is binding in the coronal canal. In this case, one should star with a wider (0.04or0.06 taper) instrument or gates Glidden drill to free up the canal so that the instrument may reach the mid-and apical canal. This would be the beginning of step-down preparation. Buchanan also emphasized the importance of removing all pulp remnants before shaping begins to ensure that this tissue does not "pile up" at the constriction and impedes full cleaning and shaping to that point ${ }^{4}$.

MODIFIED TECHNIQUE: There have been a number of modifications of the step-down technique since it was first promulgated. One of the most recent was by Ruddle. Following complete access, he suggested that clinicians "face-off" the orifices with an appropriately sized gates-Glidden drill. This creates a smooth guide path to facilitate the placement of subsequent instruments. Certain canal systems contain deep divisions and may be initially opened at their coronal ends with Micro Openers (Dentsply Maillefer; Tulsa, Okla ${ }^{4}$.).

Exploration of this portion of the canal will confirm straight-line access, cross-sectional diameter, and root canal system anatomy. Files are used serially to flare the canal until sufficient space is generated to safely introduce either Gates-Glidden or nickel titanium rotary shaping files ${ }^{4}$.

Frequent irrigation with sodium hypochlorite and recapitulation with a No.10 file will discourage canal blockage and move debris into solution, where it can be liberated from the root canal system. One way to accomplish pre-enlargement of the canal is with Gates-Glidden drill No.1 and carry each larger instrument short of the previous instrument to promote a smooth, flowing, and tapered preparation. Frequent irrigation with sodium hypochlorite and recapitulation with a small clearing file to prevent blockage in that order ${ }^{4}$.

Following pre-enlargement, Ruddle believes in negotiating the apical one-third last, establishing patency, and confirming working length. He then recommends finishing the apical zone so that there is a smooth uniform taper from the orifice level to the radiographic terminus. He emphasized that a variety of instruments may be used to create the "deep shape." If the clinician chooses 0.02 tapered files to "finish" the apical one -third, Ruddle uses a concept he calls "Gauging and Tuning". "Gauging" is knowing the cross-sectional diameter of the foramen that is confirmed by the size of instrument that "snugs in "at working length. "Tuning" is ensuring that each sequentially larger instrument uniformly backs out of the canal $0.5 \mathrm{~mm}^{4}$.

Gauging and tuning with ISO 0.02 tapered instruments is a technique directed toward confirming a uniform taper in the apical one third of the root canal preparation. The clinician begins to gauge the most apical cross-sectional diameter of the canal when a hand or rotary instrument feels snug at the terminus and resists further apical travel. To prove that the diameter of this file at length represents the true size of the foramen, the clinician must tune ${ }^{4}$.

Tuning is the clinical activity of recapitulating through a series of successively larger instruments and working them until they are observed to back out of the canal in a uniform way. The interval of back out should not be greater then $0.5 \mathrm{~mm}$. The file that begins to feel snug at length represents the true, most apical, cross-sectional diameter of the canal if each progressively larger instrument uniformly backs out of the canal in $0.5 \mathrm{~mm}$ intervals. Gauging and tuning verify deep shaping of the apical third of the canal ${ }^{4}$. 
The benefits of first pre-enlarging the coronal two-thirds of the canal are;

1) Pre-enlargement gives the clinician better tactile control when directing small, precurved negotiating files into the delicate apical third microanatomy. Early coronal two-thirds enlargement removes restrictive dentin and reduces significant pressure from the more coronal cutting flutes of any type file. pre- enlarging the coronal two thirds of a canal allows a precurved file to be more easily inserted, freely passed through areas once occupied by canyons of restrictive dentin, and gently finessed around pathways of curvature common to the apical third 4 .

2) Pre enlarged canals hold a greater volume of irrigant that serves to enhance cleaning. Narrow, more restrictive preparations are dangerous as files work in virtually dry canals. A pre-flared canal exhibits shape and holds a greater volume of warm irrigant, which accelerates the apical and lateral dissolution of pulp tissue. Early coronal enlargement increases the working time for the penetration and circulation of irrigant through all aspects of the root canal system ${ }^{4}$.

3) Pre enlarged and tapered canals dramatically promote removal of dentin mud. a preflared canal increases the volume of irrigant and provides an improved pathway for liberating dentin mud4.

4) Pre-enlargement decreases post treatment problems. because the bulk of the pulp tissue and bacteria and their endotoxins (when present) have been removed. Passing files through a cleaned, pre-enlarged preparation equates to less debris inadvertently inoculated periapically. Passing files through debris laden and infected canals has the potential to push more irritants into the periapical area, thereby causing more postoperative exacerbations ${ }^{4}$.

5) Pre-enlargement procedures improve identifying the foramen. A pre-enlarged canal passively accepts a larger file into the apical one third where its terminal extent is easier to visualize radiographically. Electronic apex locators are more reliable when used in pre-enlarged canals because instruments are more likely to contact dentin as they approach the apical foramen. When the clinician does establish a working length, it will be more accurate as it occurs after a more direct path to the terminus has been established 4 .

\section{ROTARY SHAPING GUIDELINES FOR ALL ROTARY SYSTEMS IN THE MANAGEMENT OF CURVED CANALS.}

1) Straight-line access. Straight-line access is best determined by observing whether the handle position of the files is "on" or "off" the long axis of the root, rotary-shaping techniques may commence.

2) Cross-sectional diameter. The cross-sectional diameter of a canal needs to be confirmed with hand instruments before introducing a rotary-shaping file. Sufficient space must exist to accommodate and guide the noncutting or partially active tip of a rotary instrument. for example, if a canal has been scouted to within 2 to $3 \mathrm{~mm}$ of anticipated working length with no 10 and no 150.02 tapered files then more space exists coronal to their tips than files numeric names suggest. a no 10 file and no 15 file have $16 \mathrm{~mm}$ of 
cutting flutes, and their D16 diameters are 0.42 and $0.47 \mathrm{~mm}$ respectively. These small instruments provide opening for the use of rotary instruments.

3) Root canal anatomy. Root canal anatomy may be categorized into five commonly encountered anatomic forms. Scouter files provide critical information regarding the curvature, recurvature or dilacerations of a canal. Further, before introducing rotary instruments, clinicians need to know if a single canal divides or if two canals within a root merge along their length. Generally canals exhibiting these anatomic forms are not appropriate for NiTi rotary use. Scouter files are not just measuring wires; rather, they need to be thought of as providing essential reconnaissance information before initiating shaping procedures.

4) Speed and sequencing. All rotary instruments perform optimally with less breakage when used in gear reduction electric motors that automatically adjust the torque specific for any given file used. Rotary instruments need to be used according to the guidelines gathered from market experience. To reduce the potential for breakage, rotary-shaping files are best used starting with the larger Do diameters or tapers and then proceeding to the smaller sized instruments. By using the instruments in a crowndown fashion, the area of the file engaging the canal is minimized, thereby reducing the torsional stress exerted on the instrument. NiTi rotary shaping files with variable Do diameters tend to engage and cut dentin toward their terminal cutting blades. However, NiTi rotary shaping files that have fixed Do diameters cut dentin toward their more shank side cutting flutes (i.e., toward the strongest portion of the instrument). Using files serially from large to small avoids dangerous taper lock that results when an instrument engages dentin over its full length of cutting blades.

5) Lubrication and the "light touch." to reduce the risk of breakage, rotary-shaping instruments are always used in presence of a lubricating irrigant. Pulp chambers should be filled brimful with sodium hypochlorite or a chelator to reduce friction between the instrument and the wall of the canal. Rotary instruments should be used passively within the canal, and their use should be continued as long as they move easily. As stated previously, the desired pressure on an instrument should be equivalent to the pressure used when writing with a sharp lead pencil.

\section{RECENT ADVANCEMENTS}

The new wave one niti file system from dentsply malliefer is a single-use, single-file system to shape the root canal completely from start to finish $\left({ }^{2}, 10\right)$. In most cases, the technique only requires one hand file, followed by one waveone file to shape the canal completely .

The specially designed NiTi files work in a similar but reverse "balance force" action using a preprogrammed motor to move the files in a back and forth motion "reciprocal motion". The files are manufactured using M-Wire technology, improving strength and resistance to cyclic fatigue by up to nearly four times in comparison with other

brands of rotary NiTi files.(11) All brands of NiTi files can be used with the wave one motor, as it has additional functions for continuous rotation .However as waveone files have their own unique reverse design, they can Only be used with wave one motor with its reverse reciprocating function.At present, there are three files in the Wave0ne single-file reciprocating system available in lengths of 21,25 and $31 \mathrm{~mm}$. 
1. The WaveOne Small file is used in fine canals. The tip size is ISO 21 with a continuous taper of $6 \%$.

2. The WaveOne Primary file is used in the majority of canals. The tip size is ISO 25 with an apical taper of $8 \%$ that reduces towards the coronal end.

3. The WaveOne Large file is used in large canals. The tip size is ISO 40 with an apical taper of $8 \%$ that reduces towards the coronal end.

The canal-centring ability of Wave One(12), remaining canal wall thickness after instrumentation with Wave One(13), final shape versus initial shape of the canal with WaveOne (14) the results of these studies suggest that Wave One single reciprocating files are comparable in performance to all the major leading brands of NiTi files that operate in continuous rotation.

CONCLUSION: Treatment of curved canals actually should be the same as treatment of straight canals.

The only difference is that with curved canals there is less margin for error. It is a clinical truism that if simple cases are treated the same way as difficult cases eventually even difficult cases will become simpler. ${ }^{1}$

\section{REFERENCES}

1. Clark's Clinical dentistry Volume 1: James W. Clark ; chapter in endodontics. Harper and Row, 1998.

2. Herbert Schilder: Cleaning and Shaping the root canal system, Dent Clin North Am 18(2); 269, 1974.

3. Franklin S.Weine: Intracanal Treatment Procedures, Basic and Advanced Topics.Endodontic therapy. Fifth Edition.Mosby.1972:164-239.

4. John I. Ingle et al: Endodontic Cavity Preparation. Endodontics Fifth Edition.B.C.Decker, Elsevier.2002: p 405-570.

5. A.W.K.Chan and G.S.P.Cheung. A Comparison of stainless steel and nickel titanium K-files in curved root canals.IEJ 1996; 29: 370-375.

6. Philip J.Hankins and Mahmoud E.ElDeeb: An Evaluation of the Canal Master, BalancedForce and Step-Back Techniques.JOE1996; 22(3):123-130.

7. Benjamin Briseno Marroquin. An Anti-Zipping Preparation System (Method and Instrument) for Curved Root Canals: A Preliminary Report.J.Endod.1996; 22(2):85-89.

8. Gary S.P.Cheung and Alex W.K.Chan. An in Vitro Comparison of the Excalibur Handpiece and Hand Instrumentation in Curved Root canals: J.Endod.1996; 22(3):131-136.

9. Clifford J.Ruddle: Cleaning and Shaping of the Root Canal System Cohen S, Burns R, editors; Pathways of the pulp, $8^{\text {th }}$ edition .St.Louis: Mosby 2002:p231-291.

10. West JD.Endodontic predictability-“Restore or remove: how do I choose?" In: cohen M ed. Interdisciplinary treatment planning :Principles, Design, implementation. Quintessence publishing Co.,2008:123-64.

11. Johnson E, Lloyd A ,Kuttler S,Namerow K .Comparison between a novel nickel titanium alloy and 508 nitinol on the cyclic fatigue life of profile 25/.04 rotary instruments.J E ndod 2008:34(11);1406-9. 
12. Kuttler S, Bonilla C, Perez R, Hardigan P. Evaluation of remainingcanal wall thickness and center ability after instrumentation with a new reciprocating system. 2011a. In press.

13. Webber J, Kuttler S, Bonilla C, Perez R, Hardigan P. Evaluation of remaining canal wall thickness and center ability after instrumentation with WaveOne reciprocating system vs rotary BioRace NiTi system. 2011b. In press.

14. Pertot W, Machtou M, Kuttler S, Bonilla C, Perez, R, Hardigan P. Evaluation of remaining canal wall thickness and center ability after instrumentation with WaveOne reciprocating system vs Revo-S rotary NiTi system. 2011. In press.

15. The wave one single file reciprocating system: Authors-Dr.julian Webber, Drs Pierre Machtou \& Wilhelm Pertot, France; Drs Sergio Kuttler, Clifford Ruddle \& John West, USA

NICKEL TITANIUM FILES CHRONOLOGY OF NICKEL-TITANIUM USE IN ENDODONTICS

\begin{tabular}{lllll}
\hline & Year & Cross-section & Taper & Tip \\
\hline NT engine & 1991 & Modified H file & 02 & Pilot \\
Light speed & 1992 & U file & 00 & Pilot \\
Mity roto & 1993 & U file & 02 & Pilot \\
Profile & 1993 & U file & $02-06$ & Pilot \\
Orifice shaper & 1993 & U file & $05-08$ & Pilot \\
Power R & 1994 & U file & $02-06$ & Pilot \\
Quantec & 1996 & Modified Kfile & $02-12$ & Various \\
GT rotary & 1998 & U file & $04 / 06-12$ & Pilot \\
Hero 642 & 1999 & Modified H file & $02-06$ & Modified active \\
RaCE & 1999 & Modified K file & $02-10$ & Pilot \\
Flex master & 2000 & Modified K file & $02-06$ & Modified active \\
Pro Taper & 2001 & Modified K file & Multiple/rever & Modified active \\
& & & se & \\
K3 & 2001 & Modified K file & $02-10$ & Pilot \\
Endostar & 2001 & Modified K file & $02-10$ & Pilot \\
NiTi-tee & 2002 & Modified S file & $02-12$ & Pilot \\
K2 & 2002 & Modified uni file & $02-08$ & Pilot \\
M file & 2003 & Modified K file & $02-06$ & Pilot \\
\hline
\end{tabular}

